\title{
Pancreatitis autoinmune: Experiencia clínica y revisión de la literatura
}

\author{
SERGIO MUÑOZ C. ${ }^{1}$, CARLA MANCILLA A. ${ }^{1}$, \\ LEONOR MOYANO S. ${ }^{2}$, CECILIA CASTILLO T. ${ }^{3,4}$, RICARDO ROSSI F. ${ }^{3}$, \\ JAVIER BRAHM B. ${ }^{1}$, ZOLTÁN BERGER F. ${ }^{1,5}$
}

\section{Autoimmune pancreatitis. Report of 10 cases}

Background: Autoimmune pancreatitis is a special form of chronic pancreatitis, more common in men and usually presenting as obstructive jaundice or abdominal pain. It may be associated with other immunological disorders and sometimes it is possible to find positive serological markers. Typical images show pancreatic enlargement with focal or diffuse stenosis of the pancreatic duct but sometimes it presents as a focal pancreatic mass, that is difficult to differentiate from pancreatic carcinoma. Aim: To report ten cases of autoimmune pancreatitis. Material and Methods: Retrospective review of clinical records of 10 patients aged 26 to 56 years (six males) with autoimmune pancreatitis. Results: The clinical presentation was obstructive jaundice in six cases, acute pancreatitis in two, persistent increase in serum amylase and lipase in one, and permanent abdominal pain and weight loss in one. On imaging studies, a circumscribed mass was found in six patients. An endoscopic retrograde colangiopancreatography was performed in four patients showing an abnormal pancreatic duct in all. Six patients were operated and tissue for pathological study was obtained in five, showing inflammatory infiltration. Five patients were treated with steroids with a good clinical response. Conclusions: Autoimmune pancreatitis must be borne in mind in the differential diagnosis of pancreatic lesions.

(Rev Med Chile 2010; 138: 295-302).

Key words: Autoimmunity; Pancreatitis, chronic; Prednisone.

'Sección de Gastroenterología,
Departamento de
Medicina Interna.
${ }^{2}$ Departamento de
Anatomía Patológica.
${ }^{3}$ Departamento de Cirugía.
Hospital Clínico
Universidad de Chile.
${ }^{4}$ Unidad de Endoscopia y En-
dosonografía, Clínica Alemana
de Santiago.
${ }^{5}$ Clínica Dávila.
Recibido el 15 de abril
de 2009 , aceptado el 4 de
febrero de 2010.
Correspondencia:
Dr. Zoltán Berger F
Sección de Gastroenterología,
Departamento de Medicina
Interna
Hospital Clínico
Universidad de Chile.
Santos Dumont 999.
Fono: 9788350
Fax: 9788349.
E-mail:
berger.zoltan@gmail.com

L a pancreatitis autoinmune (PAI) fue descrita por una de las figuras más grandes de la pancreatología moderna, Henri Sarles, en $1961^{1}$, denominándose inicialmente pancreatitis inflamatoria y posteriormente pancreatitis esclerosante, pancreatitis linfoplasmocitaria y finalmente PAI por Yoshida, en $1995^{2}$. Es una entidad que comparte características clínicas y morfológicas de pancreatitis aguda y crónica. Su forma de presentación es variada, observándose con cierta frecuencia una masa circunscrita al páncreas, asociación con otras patologías autoinmunes o hallazgos serológicos positivos. En muchos casos resulta difícil descartar el cáncer de páncreas y finalmente sólo el estudio histológico de la pieza quirúrgica puede comprobar su diagnóstico ${ }^{3-6}$.
En el último tiempo se han hecho esfuerzos por buscar métodos diagnósticos con el objetivo de evitar la cirugía y tratar la enfermedad de manera conservadora. En pacientes con alto nivel de sospecha se puede obtener histología por punción guiada por tomografía o endosonografía $a^{7,8}$. Además, recientemente se han descrito en PAI cambios histológicos en la papila mayor duodenal (papilitis autoinmune) que podrían contribuir al diagnóstico a través de la biopsia endoscópica de la papila, ${ }^{9,10}$.

Las experiencias en el mundo entero son crecientes con esta patología. La Asociación Japonesa de Pancreatología, la Clínica Mayo y otras entidades han elaborado nuevos criterios diagnósticos ${ }^{11-14}$. 
El objetivo de esta comunicación es presentar la experiencia acumulada en nuestros centros con un grupo de pacientes con diagnóstico de PAI, describiendo sus características y evaluando el tratamiento efectuado y la evolución de la enfermedad.

\section{Pacientes y Métodos}

Se revisaron retrospectivamente los antecedentes de 10 pacientes con diagnóstico de PAI, atendidos entre los años 1999 y 2007 en el Hospital Clínico de la Universidad de Chile y en la Clínica Dávila.

Se registraron características demográficas, forma de presentación, asociación con otras patologías autoinmunes, estudios serológicos, hallazgos imagenológicos en la tomografía axial computada (TAC), resonancia magnética (RM) y colangiopancreatografía retrógrada endoscópica (CPRE), hallazgos quirúrgicos e histológicos, evolución clínica y respuesta al tratamiento.

\section{Resultados}

De los diez pacientes, seis eran hombres y cuatro mujeres, con edad promedio 44 años (rango 26-56) (Tabla 1).

La presentación clínica más frecuente fue la ictericia obstructiva en seis casos, seguida por pancreatitis aguda en dos (una de ellas recurrente), elevación persistente de amilasa y lipasa en un caso y dolor abdominal con baja de peso en otro. Otras enfermedades autoinmunes asociadas a PAI se observaron en 5 casos, siendo de particular interés la colangitis esclerosante, en dos casos. Una paciente presentaba múltiples afecciones autoinmunes, otra tenía una artritis reumatoide y otro paciente desarrolló posteriormente una fibrosis retroperitoneal. Uno de los pacientes presentaba infección por virus de la hepatitis $\mathrm{C}$ (VHC).

La determinación de elastasa fecal se realizó en tres pacientes, siendo compatible con insuficiencia pancreática exocrina severa en ellos (elastasa fecal $<100 \mu \mathrm{g} / \mathrm{g}$ ). La determinación de $\mathrm{IgG}_{4}$ en plasma se pudo realizar sólo en un caso y fue normal. Los auto-anticuerpos inespecíficos examinados fueron negativos, con excepción del antimúsculo liso (ASMA) positivo en la paciente con múltiples afecciones autoinmunes.

En el estudio de imágenes se encontró una masa circunscrita en la TAC, RM o ambas en seis pacientes, en cinco de ellos en la cabeza y en uno en la cola del páncreas. A diferencia de los hallazgos típicos en cáncer de páncreas, el conducto pancreático proximal a la masa era normal o sólo mínimamente dilatado. En dos casos se encontró un aumento difuso de volumen del páncreas (Tabla 2).

\section{Tabla 1. Características clínicas de 10 pacientes con pancreatitis autoinmune}

\begin{tabular}{|cccll|}
\hline Paciente & Sexo & $\begin{array}{c}\text { Edad al } \\
\text { diagnóstico } \\
\text { (años) }\end{array}$ & Presentación clínica & $\begin{array}{l}\text { Otras enfermedades } \\
\text { autoinmunes }\end{array}$ \\
\hline 1 & M & 52 & Ictericia obstructiva & Colangitis esclerosante \\
\hline 2 & M & 26 & Pancreatitis aguda & No \\
\hline 3 & M & 55 & Ictericia obstructiva & Colangitis esclerosante \\
\hline 4 & M & 27 & Ictericia obstructiva & No \\
\hline 5 & F & 56 & Dolor abdominal + baja de peso & No \\
\hline 6 & F & 44 & Elevación persistente amilasa y lipasa & CBP, Sjögren, CREST, AR, Hipotiroidismo \\
\hline 7 & F & 50 & Pancreatitis aguda recurrente & AR, Vasculitis \\
\hline 8 & M & 45 & Ictericia obstructiva & VHC \\
\hline 9 & M & 55 & Ictericia obstructiva & Fibrosis retroperitoneal \\
10 & F & 30 & Ictericia obstructiva & No \\
\hline
\end{tabular}

(CBP: Cirrosis biliar primaria; AR: Artritis reumatoidea; VHC: Virus hepatitis C; CREST: calcinosis, Raynaud, esofagopatía, esclerodactilia, telangiectasia). 
Tabla 2. Características imagenológicas, histológicas y tratamiento en 10 pacientes con PAI

\begin{tabular}{|c|c|c|c|c|c|}
\hline Paciente & TAC & $\mathbf{R M}$ & CPRE & Histología & Tratamiento \\
\hline 1 & $\begin{array}{l}\text { Masa en la } \\
\text { cabeza del } \\
\text { páncreas }\end{array}$ & $\begin{array}{l}\text { Irregularidad } \\
\text { conducto principal } \\
\text { sugerente de colan- } \\
\text { gitis esclerosante }\end{array}$ & No & $\begin{array}{l}\text { Pancreatitis periduc- } \\
\text { tal y lobulillar } \\
\text { Papilitis } \\
\text { linfoplasmocitaria }\end{array}$ & $\begin{array}{l}\text { Pancreatoduode- } \\
\text { nectomía } \\
\text { AUDC + Prednisona }\end{array}$ \\
\hline 2 & No & $\begin{array}{l}\text { Páncreas con } \\
\text { aumento de señal } \\
\text { heterogéneo más } \\
\text { edema del surco } \\
\text { pancreatoduodenal }\end{array}$ & No & No & Prednisona \\
\hline 3 & $\begin{array}{l}\text { Masa en la } \\
\text { cabeza del } \\
\text { páncreas }\end{array}$ & $\begin{array}{l}\text { Estenosis arrosaria- } \\
\text { da de los conductos } \\
\text { biliares e intrapan- } \\
\text { creáticos }\end{array}$ & $\begin{array}{l}\text { Estenosis colé- } \\
\text { doco intrapan- } \\
\text { creático. }\end{array}$ & $\begin{array}{l}\text { Fibrosis e inflama- } \\
\text { ción linfoplasmo- } \\
\text { citaria }\end{array}$ & $\begin{array}{l}\text { Stent biliar Pancrea- } \\
\text { toduodenectomía } \\
\text { Prednisona + AUDC }\end{array}$ \\
\hline 4 & Páncreas normal & $\begin{array}{l}\text { Estenosis filiforme } \\
\text { del Wirsung en } \\
\text { cabeza del páncreas } \\
\text { sin dilatación cuer- } \\
\text { po y cola }\end{array}$ & $\begin{array}{l}\text { Estenosis del } \\
\text { colédoco intra- } \\
\text { pancreático } \\
\text {. }\end{array}$ & $\begin{array}{l}\text { Pancreatitis crónica } \\
\text { con fibrosis e } \\
\text { infiltrado mononu- } \\
\text { clear periductal y } \\
\text { lobulillar } \\
\text { Papilitis severa }\end{array}$ & $\begin{array}{l}\text { Stent biliar Pancrea- } \\
\text { toduodenectomía }\end{array}$ \\
\hline 5 & $\begin{array}{l}\text { Masa cola } \\
\text { páncreas y } \\
\text { adenopatías }\end{array}$ & $\begin{array}{l}\text { Masa cola páncreas } \\
\text { y adenopatías }\end{array}$ & No & $\begin{array}{l}\text { Pancreatitis crónica } \\
\text { con fibrosis e } \\
\text { infiltrado linfoplas- } \\
\text { mocitario }\end{array}$ & $\begin{array}{l}\text { Pancreatectomía } \\
\text { distal }\end{array}$ \\
\hline 6 & No & No & $\begin{array}{l}\text { Múltiples } \\
\text { estenosis del } \\
\text { Wirsung con } \\
\text { vía biliar normal }\end{array}$ & No & AUDC + Prednisona \\
\hline 7 & $\begin{array}{l}\text { Aumento densi- } \\
\text { dad páncreas }\end{array}$ & No & $\begin{array}{l}\text { Estenosis focal } \\
\text { del Wirsung con } \\
\text { vía biliar normal }\end{array}$ & No & Prednisona \\
\hline 8 & $\begin{array}{l}\text { Masa en la } \\
\text { cabeza del } \\
\text { páncreas }\end{array}$ & No & No & $\begin{array}{l}\text { Pancreatitis linfo- } \\
\text { plasmocitaria }\end{array}$ & $\begin{array}{l}\text { Pancreatoduode- } \\
\text { nectomía }\end{array}$ \\
\hline 9 & $\begin{array}{l}\text { Masa en la } \\
\text { cabeza del } \\
\text { páncreas }\end{array}$ & No & No & $\begin{array}{l}\text { Papilitis linfoplas- } \\
\text { mocitaria }\end{array}$ & $\begin{array}{l}\text { Laparotomía explo- } \\
\text { radora }+ \\
\text { Prednisona }\end{array}$ \\
\hline 10 & $\begin{array}{l}\text { Masa en la } \\
\text { cabeza del } \\
\text { páncreas }\end{array}$ & $\begin{array}{l}\text { Masa en la cabeza } \\
\text { del páncreas }\end{array}$ & No & $\begin{array}{l}\text { Papilitis linfoplas- } \\
\text { mocitaria }\end{array}$ & Prednisona \\
\hline
\end{tabular}

(AUDC: Ácido ursodeoxicólico).

Se realizó CPRE en cuatro pacientes. El conducto pancreático presentó cambios patológicos en todos ellos: estenosis sospechosa de neoplasia, con mínima dilatación proximal en dos casos (Figura 1); estenosis focal única en otro y múltiples estenosis en el cuarto paciente. Además de los cambios descritos en el conducto pancreático, la vía biliar presentó también alteraciones morfológicas en dos de los cuatro pacientes, con estenosis del colédoco intrapancreático y dilatación de la vía biliar proximal, colocándose endoprótesis biliar en ambos casos. En uno de ellos se observaron también múltiples estrecheces de la vía biliar proximal, compatibles con colangitis esclerosan- 


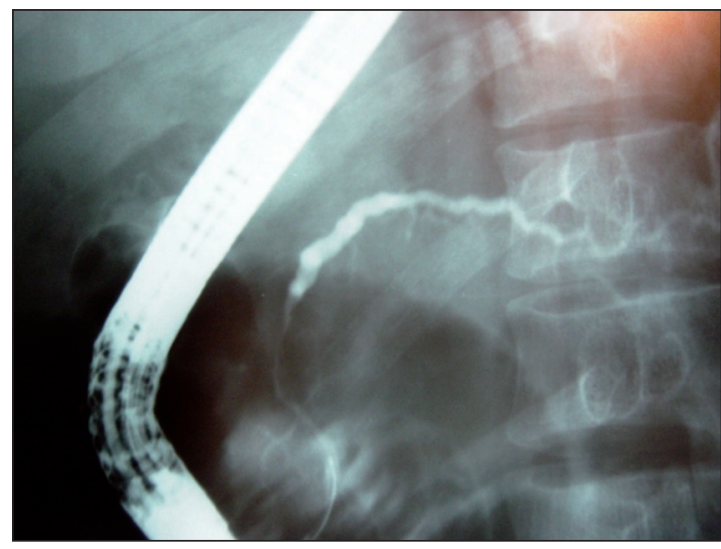

Figura 1. Pancreatografía endoscópica mostrando estenosis filiforme del conducto de Wirsung en la cabeza del páncreas con mínima dilatación hacia cuerpo y cola de la glándula.

te. La vía biliar fue normal en dos de los cuatro pacientes.

Se intervinieron quirúrgicamente seis pacientes por sospecha de cáncer de páncreas, realizándose pancreatoduodenectomía con preservación del píloro en cuatro de ellos, resección distal de páncreas con esplenectomía en uno y sólo laparotomía exploradora en otro. Por consiguiente, se obtuvo un estudio histopatológico de la pieza quirúrgica en 5 casos, demostrándose infiltrado linfoplasmocitario ductal, periductal y perivascular, flebitis obliterativa y fibrosis, sin calcificaciones (Figuras 2 y 3 ).

Revisando las piezas quirúrgicas en forma retrospectiva, se pudo evaluar la papila en dos casos, encontrándose en ambos una infiltración linfoplasmocitaria marcada, muy similar a las alteraciones pancreáticas. Además se observó la misma alteración en dos biopsias de papila tomadas por vía endoscópica.

En uno de los pacientes se sospechó un cáncer pancreático en el contexto de ictericia obstructiva y masa en la cabeza del páncreas, considerándose irresecable en la laparotomía exploradora. Varios meses más tarde se observó una disminución espontánea de la ictericia y de la masa, procediéndose a la biopsia endoscópica de la papila, que reveló papilitis linfoplasmocitaria. Se inició tratamiento con prednisona, con desaparición completa de la masa en la TAC realizada en la cuarta semana de tratamiento y recuperación clínica completa.

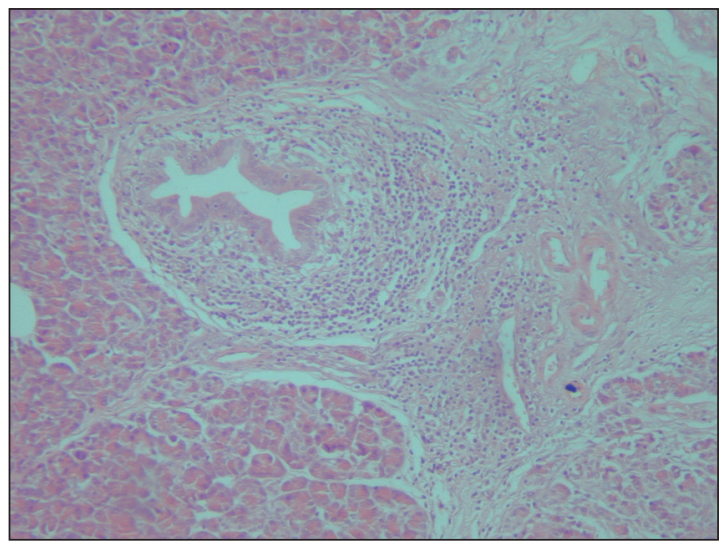

Figura 2. Corte histológico del páncreas con infiltrado linfoplasmacitario periductal, fibrosis periductal, con estructura acinar conservada y sin fibrosis. (HE 100x).

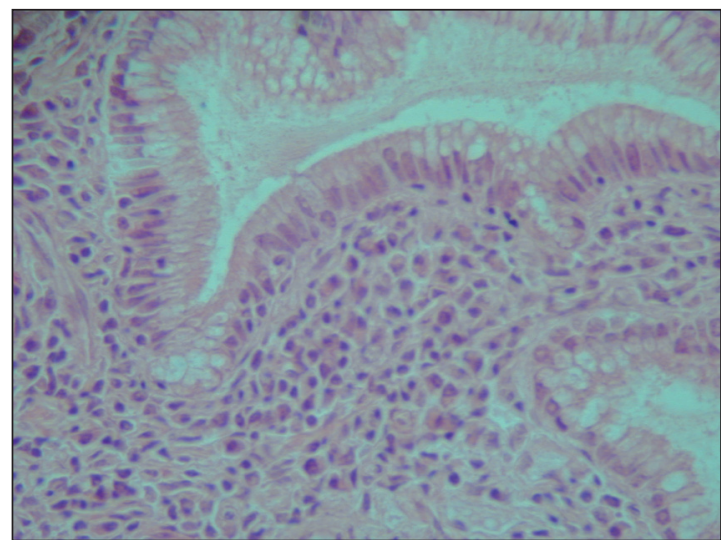

Figura 3. Corte histológico de Papila mayor con infiltrado periductal rico en células plasmáticas. (HE 400x).

$\mathrm{Al}$ establecer el diagnóstico, otros cuatro pacientes recibieron tratamiento esteroidal, todos ellos con respuesta clínica e imagenológica parcial o completa dentro de cuatro semanas. La dosis de prednisona fue de $30 \mathrm{mg}$ /día por 4 semanas, que se disminuyó gradualmente hasta suspender al tercer mes. Tres pacientes se encuentran recibiendo ácido ursodeoxicólico (AUDC), 2 por colangitis esclerosante y una por cirrosis biliar primaria conocida y tratada ya previamente.

No se ha observado recurrencia durante el seguimiento (rango 6 meses a 10 años), tanto en los pacientes operados como en los sometidos a tratamiento conservador. 


\section{Discusión}

La PAI no es una entidad homogénea, pudiendo comprometer difusamente el páncreas o producir lesiones focales. Clínicamente puede manifestarse en forma pseudotumoral, en forma de pancreatitis crónica con insuficiencia exocrina, o más infrecuentemente como pancreatitis aguda con alza enzimática ${ }^{3-6,15}$.

La PAI se presenta en ambos sexos, siendo dos veces más común en hombres que en mujeres. La edad de presentación más frecuente es entre los 50 y 60 años con un rango que va desde los 30 a los 80 años $^{3-6}$. En este pequeño grupo de pacientes encontramos un leve predominio en hombres (6:4) con un amplio rango en la edad de presentación.

Su etiología y patogenia son poco conocidas. Mecanismos inmunológicos estarían involucrados, siendo el sello histológico de la enfermedad el infiltrado linfoplasmocitario de linfocitos $\mathrm{T} \mathrm{CD}^{+} \mathrm{y}$ $\mathrm{CD}^{+}{ }^{+}$que sintetizan interferón gamma, activando la cascada inflamatoria ${ }^{3-7,15}$.

La hipergammaglobulinemia es frecuente a expensas de la subclase $\operatorname{IgG}_{4}$, marcador de esta enfermedad, que tiene además elevada especificidad. La sensibilidad reportada es muy variable en las distintas series, con rangos de 73 a $90 \%$ para valores de $\mathrm{IgG}_{4}$ sobre $135 \mathrm{mg} / \mathrm{dl}$. Sin embargo, en occidente, el rendimiento de este test parece ser mucho menor ${ }^{16,17}$. En nuestra serie sólo fue posible determinar marcadores inmunológicos específicos en una paciente, donde la $\mathrm{IgG}_{4}$ resultó normal. La positividad de ASMA en aquella paciente con múltiples afecciones autoinmunes, hace compleja su interpretación como marcador de la enfermedad pancreática.

Por otra parte, los marcadores serológicos más específicos de la enfermedad serían los anticuerpos antianhidrasa carbónica II y antilactoferrina ${ }^{3-5,12,15}$.

Para algunos autores, la PAI sería una enfermedad sistémica, dada su asociación con otras patologías autoinmunes. La más frecuente es el síndrome de Sjögren, estando también descrita la asociación con artritis reumatoide, hipotiroidismo, colitis ulcerosa, colangitis esclerosante, cirrosis biliar primaria (CBP) y pseudotumores inflamatorios (hepáticos, renales, pulmonares) ) $^{15,18,19}$. En nuestra serie, la asociación con otras enfermedades autoinmunes fue también frecuente siendo de particular interés la colangitis esclerosante, que parece ser una forma especial de la enfermedad, por su buena respuesta al tratamiento esteroidal, lo que coincide con esta experiencia ${ }^{20}$. Respecto al paciente con infección por virus hepatitis $\mathrm{C}$ (VHC), es conocido que ésta puede asociarse a enfermedades autoinmunes, sin embargo, no hemos encontrado datos publicados de asociación entre VHC y PAI.

La literatura describe como forma de presentación ictericia indolora por crecimiento de la cabeza del páncreas (63\%), dolor abdominal (35\%), diabetes mellitus (50\%), baja de peso y, más raramente, pancreatitis aguda ${ }^{3-6,15,21}$. Nosotros observamos seis casos de ictericia obstructiva, dos casos de pancreatitis aguda leve (uno recurrente), uno estudiado por alza persistente de amilasa y lipasa y otro por dolor abdominal.

Respecto a las imágenes, la TAC de abdomen con compromiso pancreático difuso muestra la apariencia clásica, con aumento de tamaño del páncreas en forma de "embutido" o "salchicha", con halo periférico de hipoatenuación (edema del surco pancreatoduodenal). El compromiso de la grasa peripancreática usualmente es mínimo o ausente $e^{3-6,15}$. El compromiso pancreático focal, más común en la cabeza del páncreas, típicamente aparece en la TAC como una masa, que junto al hallazgo de estenosis difusas o largas de los ductos pancreáticos es sugerente de $\mathrm{PAI}^{3-6,15}$. Asimismo, los hallazgos a la RM incluyen masa focal o crecimiento difuso de la glándula con hiperintensidad en secuencias T2 y halo periférico hipointenso $0^{4,5,15,22}$.

Si bien el diagnóstico definitivo de la PAI es histológico ${ }^{4,5,15,22}$, el aporte de las imágenes es cada vez mayor para reconocer esta entidad. Resulta particularmente difícil distinguirla del cáncer de páncreas en la forma que se manifiesta como masa circunscrita. En estos casos, la ausencia de dilatación del Wirsung es un argumento en contra del cáncer. En caso de compromiso difuso, el hallazgo imagenológico que debe hacer sospechar PAI, es la disociación entre un parénquima con alteraciones compatibles con inflamación aguda leve, sin compromiso de la grasa peripancreática, con un conducto que muestra estenosis focales compatibles con PC, pero sin dilatación.

En la CPRE se observan segmentos estenóticos del Wirsung sin dilatación significativa y sin cálculos intraductales. Otro hallazgo frecuente en la forma pseudotumoral es la estenosis del colédoco intrapancréatico ${ }^{3-6,15}$. En la endosonografía (EUS), 
el hallazgo más común es el crecimiento difuso o focal de la glándula, con parénquima difusamente hipoecogénico ${ }^{3,4,8,15}$.

En el diagnóstico diferencial entre PAI y PC, la ausencia de calcificaciones y el diámetro normal o disminuido del conducto pancreático sugieren PAI.

Por lo tanto, las alteraciones morfológicas generalmente se encuentran en una combinación inhabitual o incompatible con cualquier otro diagnóstico.

En nuestra serie, la TAC o RM mostró en seis pacientes la presencia de una masa pancreática (cinco en la cabeza y una en la cola), en dos casos encontramos aumento difuso de volumen pancreático y edema del surco pancreatoduodenal y dos fueron normales. En cuatro pacientes predominaron los cambios ductales pancreáticos o del colédoco.

En la actualidad es importante destacar el rol de la endosonografía que permite además la obtención de biopsia por punción. En nuestra serie no dispusimos de esta técnica durante el período de estudio.

En los últimos años, diferentes grupos de estudio han desarrollado distintos criterios diagnósticos ${ }^{3-6,11-14}$, que fueron reunidos por la Clínica Mayo en la clasificación HISORt del año 2006; sin embargo, por su complejidad, sugerimos los criterios simplificados de la Japan Pancreas Society (Tabla 3).

En esta experiencia presentamos diez casos,

Tabla 3. Criterios diagnósticos de pancreatitis autoinmune (modificados de la Japan Pancreas Society 2002) $^{4}$

\section{Hallazgos imagenológicos (uno requerido)}

\section{Imagen al TAC}

Crecimiento difuso pancreático

Halo periférico de hipoatenuación

Masa de hipoatenuación en cabeza del páncreas

\section{CPRE O CPRM}

Estenosis pancreática ductal segmentaria

Estenosis pancreática ductal focal

Estenosis pancreática ductal difusa

TAC: Tomografía axial computada; CPRE: Colangiopancreatografía endoscópica retrógrada; CPRM Colangiopancreatografía por resonancia magnética.

\section{Hallazgos serológicos e histológicos (uno requerido)}

\begin{tabular}{lll}
\hline Serología & Histología & Histología no gastrointestinal \\
\hline Alza $\operatorname{lgG}_{4}$ sérica & $\begin{array}{l}\text { Infiltración periductal linfoplas- } \\
\text { mocitaria o fibrosis }\end{array}$ & $\begin{array}{l}\text { Nefritis túbulointersticial con depósitos inmunes } \\
\text { dentro de la membrana basal tubular }\end{array}$ \\
$\begin{array}{l}\text { Alza IgG o gammaglobulinas } \\
\text { séricas }\end{array}$ & $\begin{array}{l}\text { Flebitis obliterativa } \\
\text { Presencia de AcALF, AcAAC-II, } \\
\text { ASMA o ANA }\end{array}$ & $\begin{array}{l}\text { Infiltración linfoplasmocitaria intersticial pulmonar } \\
\text { con aumento de las células plasmáticas } \text { IgG }_{4}{ }^{+}\end{array}$ \\
\hline
\end{tabular}

AcALF: Anticuerpos anti-lactoferrina; AcAAC-II: Anticuerpos anti-anhidrasa carbónica II; ASMA: Anticuerpos antimúsculo liso; ANA: Anticuerpos antinucleares; $\operatorname{lgG}_{4}^{+}$: Inmunoglobulina $G$ subclase $\lg _{4}$ positiva.

\section{Criterios Diagnósticos según distintos grupos ${ }^{12}$ :}

\begin{tabular}{|c|c|c|}
\hline Japan Pancreas Society & $2002 / 2006$ & Imagen + laboratorio y/o histología \\
\hline Grupo italiano & 2003 & $\begin{array}{l}\text { Histología o citología + asociación otras inmunopatías + respuesta a } \\
\text { esteroides }\end{array}$ \\
\hline Asan Medical Center Korea & 2006 & Imagen + laboratorio o histología o respuesta a esteroides \\
\hline HISORt Mayo Clinic & 2006 & $\begin{array}{l}\text { Histología + imagen o serología o compromiso otros órganos o respuesta } \\
\text { a esteroides }\end{array}$ \\
\hline
\end{tabular}


todos los cuales cumplieron criterios diagnósticos por imágenes y confirmados por histología en siete de ellos (cinco del páncreas y dos de papila) y en los tres restantes apoyados por la respuesta favorable al tratamiento esteroidal.

El principal diagnóstico diferencial, sobre todo en la forma focal de PAI, es el adenocarcinoma de la cabeza del páncreas. Además de la biopsia pancreática, la respuesta rápida al tratamiento esteroidal, con disminución del tamaño de la masa y una rápida resolución de las lesiones ductales y obstrucción biliar, puede ayudar a comprobar el diagnóstico y permite evitar la cirugía y continuar con un tratamiento conservador ${ }^{23}$. En nuestros pacientes, seis debieron ser sometidos a cirugía por imposibilidad de descartar una neoplasia, lo que no difiere de la experiencia internacional. Es importante destacar que el subdiagnóstico de PAI en lesiones focales del páncreas puede conducir a cirugías innecesarias y, en caso contrario, el diagnóstico erróneo, a no operar verdaderos cánceres.

La dosis de prednisona no está claramente definida en la literatura. Se recomienda iniciar el tratamiento con $0,5-1 \mathrm{mg} / \mathrm{kg} /$ día por algunas semanas, seguido por una disminución gradual. En nuestra experiencia utilizamos un tratamiento de aproximadamente tres meses, con buena respuesta. El rol de los agentes inmunosupresores como la azatioprina todavía no está aclarado ${ }^{3}$.

En los casos que cursan con ictericia obstructiva por estenosis del colédoco puede instalarse un stent, que puede retirarse en general a los $2 \mathrm{a}$ 3 meses $^{6}$.

En cuanto al pronóstico, en la mayoría de los pacientes la enfermedad remite, dejando algunas veces una glándula atrofiada con o sin disfunción pancreática. Se han reportado recaídas en $17 \%$ luego de la terapia esteroidal e incluso post-cirugía ${ }^{24,25}$, lo que no ha ocurrido en ninguno de nuestros pacientes hasta la fecha.

En síntesis, la PAI es un tipo especial de PC de difícil pesquisa, principalmente por falta de sospecha, la que sin embargo, está siendo reconocida con mayor frecuencia. En algunos casos es una manifestación particular de una enfermedad autoinmune más generalizada, mientras que en otros el páncreas es el único órgano comprometido. La sospecha diagnóstica surge de una combinación inhabitual de hallazgos clínicos y radiológicos.

Los hallazgos de laboratorio ( $\mathrm{IgG}_{4}$, autoanticuerpos) pueden apoyar el diagnóstico, aun- que la confirmación definitiva es histológica. Los hallazgos positivos en las muestras de la papila, podrían limitar la necesidad de la biopsia pancreática sólo a los casos dudosos. El diagnóstico no invasivo podría evitar intervenciones quirúrgicas innecesarias, ya que la PAI responde al tratamiento esteroidal. Los criterios recientemente publicados hacen posible el diagnóstico con herramientas de uso rutinario.

\section{Referencias}

1. Sarles H, Sarles Jc, Muratore R, Guien C. Chronic inflammatory sclerosis of the pancreas: an autonomous pancreatic disease? Am J Dig Dis 1961; 6: 688-98.

2. Yoshida K, Toki F, Takeuchi T, Watanabe S, Shiratori K, Hayashi N. Chronic pancreatitis caused by an autoimmune abnormality. Proposal of the concept of autoimmune pancreatitis. Dig Dis Sci 1995; 40: 1561-8.

3. Pickartz T, Mayerle J, Lerch M. Autoimmune pancreatitis. Nature Clin Pract Gastroenterol Hepatol 2007; 4: 314-23.

4. Finkelberg D, Sahani D, Deshpande V, Brugge R. Autoimmune Pancreatitis. N Engl J Med 2006; 355: 2670-6.

5. Lara L, Chari S. Autoimmune Pancreatitis. Current Gastroenterol Reports 2005; 7: 101-6.

6. Kim KP, Kim MH, Song MH, Lee SS, Seo DW, Lee SK. Autoimmune Chronic Pancreatitis. Am J Gastroenterol 2004; 99: 1605-16.

7. Muñoz S. Pancreatitis Autoinmune. Gastroenterol Latinoam 2007; 18: 183-7.

8. Salla C, Chatzipantellis P, Konstantinou P, Karoumpalis I, Pantazopoulou A, Tsiotos G. EUS-FNA Contribution in the Identification of Autoimmune Pancreatitis: A Case Report. J Pancreas 2007; 8: 598-604.

9. Kamisawa T, Tu Y, Nakajima H, Egawa N, Tsuruta K, Okamoto A. Usefulness of biopsying the major duodenal papilla to diagnose autoimmune pancreatitis: A prospective study using IgG4- immunostaining. World J Gastroenterol 2006; 12: 2031-3.

10. Sahin P, Pozsar J, Simon K, Illyes G, Laszlo F, Topa L. Autoimmune Pancreatitis Associated With ImmuneMediated Inflammation of the Papilla of Vater: Report on Two Cases. Pancreas 2004; 29: 162-6.

11. Okazaki K, Uchida K, Matsushita M, Takaoka M. How to diagnose autoimmune pancreatitis by the revised Japanese clinical criteria. J Gastroenterol 2007; 42 (Suppl 18): 32-8.

12. Kwon S, Kim MH, Choi EK. The Diagnostic Criteria for Autoimmune Chronic Pancreatitis: It is Time to Make a Consensus. Pancreas 2007; 34: 279-86. 
13. Morana G, Tapparelli M, Faccioli N, D’Onofrio M, Pozzi Mucelli R. Autoimmune Pancreatitis: Instrumental Diagnosis. JOP. 2005; 6: 102-7.

14. Chari S. Diagnosis of autoimmune pancreatitis using its five cardinal features: introducing the Mayo Clinic's HISORt criteria. J Gastroenterology 2007; 42: 39-41.

15. Okazaki K. Autoimmune Pancreatitis: Etiology, Pathogenesis, Clinical Findings and Treatment. The Japanese Experience. JOP 2005; 6 (Suppl 1): 89-96.

16. Kojima M, Sipos B, Klapper W, Frahm O, Knuth HC, Yanagisawa A, et al. Autoimmune Pancreatitis: Frecuency $\mathrm{IgG}_{4}$ Expression, and Clonality of T and B Cells. Am J Surg Pathol 2007; 31: 521-8.

17. Kamisawa T, Okamoto A, Funata N. Clinicopathological Features of Autoimmune Pancreatitis in Relation to Elevation of Serum IgG4. Pancreas 2005; 31: 28-31.

18. Ohara H, Nakazawa T, Ando T, Joh T. Systemic extrapancreatic lesions associated with autoimmune pancreatitis. J Gastroenterol 2007; 42 (Suppl18): 15-21.

19. Silverman WB. Autoimmune pancreatitis: more com- mon and important than we thought?. Gastrointest Endoscopy 2007; 65: 109-10.

20. Ghazale A, Chari ST, Zhang L, Smyrk TC, Takahashi $\mathrm{N}$, Levy MJ, et al. Immunoglobulin $\mathrm{G}_{4}$-Associated Cholangitis: Clinical Profile and Response to Therapy. Gastroenterology 2008; 134: 706-15.

21. Kawa S, Hamano H. Clinical features of autoimmune pancreatitis. J Gastroenterol 2007; 42 (Suppl 18): 9-14

22. Suda K, Takase M, Fukumura Y, Kashiwagi S. Pathology of autoimmune pancreatitis and tumor-forming pancreatitis. J Gastroenterol 2007; 42: 22-7.

23. Ito $\mathrm{T}$, Nishimori I, Inoue $\mathrm{N}$, Kawabe $\mathrm{K}$, Gibo J, Arita $\mathrm{Y}$, et al. Treatment for autoimmune pancreatitis: consensus on the treatment for patients with autoimmune pancreatitis in Japan. J Gastroenterol 2007; 42: 50-8.

24. Hirano K, Tada M, Isayama $H$, Yagioka $H$, Sasaki $T$, Kogure $\mathrm{H}$, et al. Long-term Prognosis of Autoimmune Pancreatitis without and with Corticosteroid Treatment. Gut 2007; 56: 1719-24.

25. Kamisawa T, Okamoto A. Prognosis of autoimmune pancreatitis. J Gastroenterol 2007; 42: 59-62. 